\title{
Using Affective Trajectories to Describe States of Flow in Interactive Art
}

\author{
Stephen W. Gilroy \\ Teesside University \\ Middlesbrough, UK
}

s.w.gilroy@tees.ac.uk

\author{
Marc Cavazza \\ Teesside University \\ Middlesbrough, UK
}

\author{
Maurice Benayoun \\ CiTu \\ Université Paris 1, France
}

m.o.cavazza@tees.ac.uk

\begin{abstract}
Interactive Art installations often integrate sophisticated interaction techniques with visual presentations contributing to a rich user experience. They also provide a privileged environment in which to study user experience by using the same sensing data that support interaction. In this paper, using the affective interface of an Augmented Reality Art installation, we introduce a framework relating real-time emotional data to phenomenological models of user experience, in particular the concept of Flow. We propose to analyse trajectories of affect in a continuous emotional space (Pleasure-Arousal-Dominance), to characterize user experience. Early experiments with several subjects interacting in pairs with the installation support this mapping on the basis of Flow questionnaires. This approach has potential implications for the analysis of user experience across Art and Entertainment applications.
\end{abstract}

\section{Categories and Subject Descriptors}

H5.1. Information interfaces and presentation (e.g., HCI): Multimedia Information Systems - augmented reality, evaluation.

\section{General Terms}

Measurement, Experimentation, Theory.

\section{Keywords}

Emotion, new media, entertainment, augmented reality, affective art.

\section{INTRODUCTION}

Interactive Art has recently become an area of interest for HumanComputer Interaction (HCI) at different levels, from the new interaction paradigms it could introduce, to the specific context it provides to study user experience [4] [25] [5].

Interactive Art relying on high-end HCI technologies (multimodal interfaces, virtual and augmented reality) is of particular interest since it sits at the convergence of emerging areas, including affective computing [29], aesthetic computing [15], and entertainment theory [37].

One approach to the study of Interactive Art experience consists in extending methods and protocols developed for the underlying HCI technology, e.g., studying Presence in those installations based on virtual environments. This would be justified theoretically at least in part by the conceptual proximity between various forms of 'suspension of disbelief', which are studied through concepts such as Media Presence. However, such an approach is faced with several limitations. Firstly it fails to capture aesthetic aspects as well as the affective components of the user experience. Secondly, as we have suggested as part of previous work [6], methods developed for the study of Presence, with their strong emphasis on realism, may not be adequate to the measurement of user experience when this is deliberately aimed at creating alternative realities (for instance, a world incorporating unrealistic laws of physics).

It thus seems more appropriate to evaluate the user experience using recent advances in Entertainment Psychology and Entertainment Theory, and in this paper we focus on the concept of Flow [10], which seems to hold some promise for describe user engagement in an interactive and aesthetic experience. One of the major challenges is to establish a framework relating the technical aspects of Interactive Art installations to the phenomenological concepts put forward in Entertainment Theory. Since post-hoc questionnaires of experience have shown their limitations in capturing the actual dynamics of the Interactive Art experience, we wish to derive measures of user experience from measurements related to the interaction itself.

Popper, in his theorisation of Digital Arts has suggested that in interactive digital installations, the interaction itself was a major component of a digital artwork's aesthetics [31]. In that sense, being able to capture the affective content of interaction could be a way to gain insight into the specific aesthetic experience of interactive art, and even in the long-term to address it more directly to design more engaging installations.

In this paper, we propose such a framework based on the state-ofthe-art analysis of user emotional reactions to an installation, using multimodal affective interfaces [16]. Our objective is to obtain a high-level analysis of user experience using generic emotional models and explore how the temporal analysis obtained by such models can be mapped to concepts in Entertainment Theory such as Flow.

\section{THE E-TREE: AN INTERACTIVE ARTWORK WITH MULTIMODAL AFFECTIVE SENSING}

In order to support evaluation of this framework, we have utilised an affective Interactive Art installation known as E-Tree 
(Emotional Tree) [16,17]. Based on an Augmented Reality system, this artwork provides a 3-D visual representation of an organically growing tree, reacting to user emotions and physical manipulations. (Figure 1.)

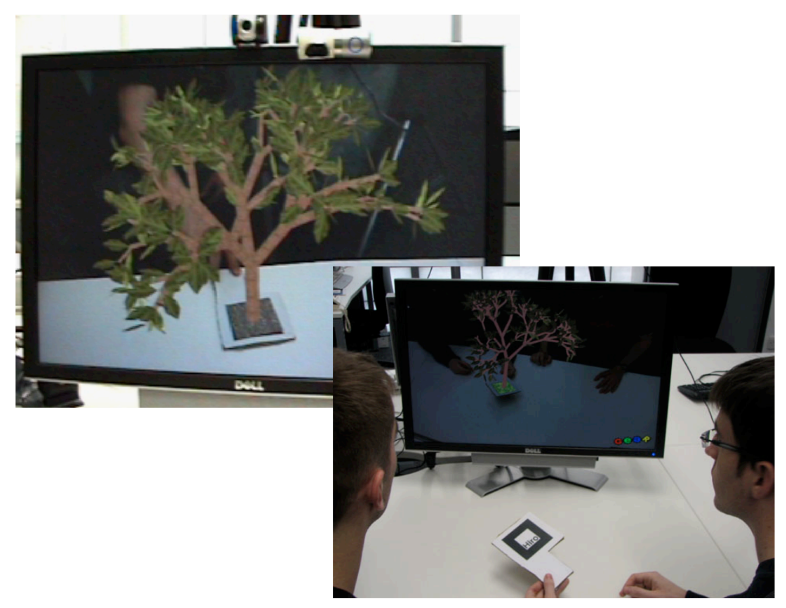

Figure 1. E-Tree affective interactive artwork.

This was developed from a brief by one of the authors (MB), as an established digital artist [18] who has previously publicly exhibited several digital artworks that have featured emotions as their main topic, such as "Emotional Traffic", "Frozen Feelings" or the "Emotion Vending Machine" [1]. The E-Tree is the continuation of his artistic research, utilising affective sensing techniques which by directly capturing the user affective states support a more explicit authoring of the installation responsiveness.

The e-Tree exploits the temporal dynamics of a growing plant to record a history of the affective interaction, which is continuously integrated into the tree's growth patterns. The manifestation of the e-Tree's empathic response is designed by the artist to convey a perceived emotional representation of the interactive experience through stylized visual metaphors. The augmented reality interface provides further support for direct interactions with the tree itself.

Other artistic systems that rely on detected emotion [34] or physiological proxies to emotions [13] as modulators of graphical effects note the connection an audience experiences when exposed to the feedback between the artwork and bodily reactions, which encourages deliberate exploration of this connection as an interactive mechanism.

The design of the E-Tree attempts to exploit this feedback, producing visualisations of the spontaneous emotional reactions of an audience, embodying monotonic, continuous properties that stimulate deliberate experimentation with the sensory apparatus through acted emotions, bodily movements and gestures, paralinguistic utterances, and speech acts. It creates a unique affective feedback loop which conspicuously supports a novel type of interactive experience.

The E-Tree expands on the affective interfaces of these systems through affective multimodal fusion, tracking in real-time a number of different interaction modalities such as speech and non-verbal behaviour (e.g., spontaneous body gestures, interest models), and combining affective interpretations into a common model of emotion. This gives a novel combination of affective affordances that also allows group interactions.

The visual metaphor of virtual plants in Interactive Art has always offered a good context for interaction and visualisation, as demonstrated in the previous works of Sommerer and Mignonneau [35], Bernhaupt et al. [3] and Watanabe [38]. (The latter two also include a specific affective interface component, although to our knowledge the e-Tree is the only installation using an explicit underlying emotional model).

\section{MODELLING REAL-TIME AFFECTIVE STATES USING A PAD MODEL}

Real-time affective interfaces require the adoption of an emotional model to serve as a formalism to represent and interpret affective input, and the e-Tree, making use of a multimodal affective interface, is no exception. The affective formalism used is the Pleasure-Arousal-Dominance (PAD) model of Mehrabian [24]. There are multiple justifications for this choice: i) dimensional models such as PAD do not impose emotional categories and are compatible with complex affective experiences, including aesthetic ones, which may not be otherwise covered by standard emotional categories. ii) dimensional models, being continuous models, support the representation of affective state over time; iii) the PAD model has been previously used to represent aesthetic appraisal of design solutions $[32,20]$; iv) the Dominance component of the PAD model is a good candidate to characterise some of the affective impact of the meta-properties of interaction: this can also be related to the work of Popper [31], according to which the interaction is an important component of the aesthetic experience of Interactive Art. We also posit that this can be an important part of identifying flow.

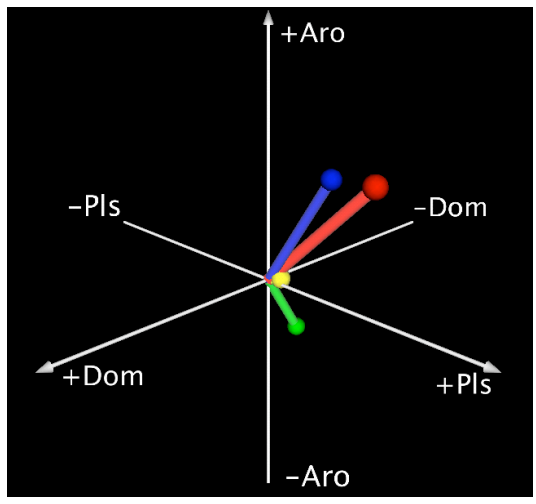

Figure 2. PAD affective space and modality vectors.

The PAD model measures emotional tendencies and response along three dimensions: pleasure-displeasure, corresponding to valence and cognitive evaluative judgements; arousal/non-arousal to levels of alertness and physical activity; and dominancesubmissiveness to the feeling of control and influence over others and surroundings. These three dimensions are sufficient for a general description of emotions. This three-dimensional affective space is shown in figure 2, together with a representative "vector" of the current emotional state (in red). The other vectors are the intermediate values from each affective input that are combined to give the overall state.

Mehrabian [24] relates how reactive behavioural tendencies can be expressed in terms of PAD values, which supports the idea of mapping interpretation of interactions during an interactive 
experience to a PAD representation. Note that these tendencies are manifest are posited to be universal, so should also have a bearing on both spontaneous emotional reaction and the nature of individual deliberate interactive exploration.

\section{AFFECTIVE INTERACTION WITH THE \\ E-TREE}

The E-Tree is not intended to exhibit purely positive feedback reinforcement - in general, all affective inputs are equally "valid", represented in the characteristic visuals of the tree and no region of PAD emotional space is "preferred". The tree visualisations are intended to have an interpretable anthropic quality, so that an audience could conceivably have only negative reactions to visualisations of previous negative reactions, and vice versa. However, that would discount the exploratory affordances of the system, which as mentioned earlier, are an integral part of such an interactive artwork.

In general, not engaging with the sensory apparatus of the affective system would result in a neutral affective interpretation, interpreted by the artwork. However, as bodily user movements and audience presence are inputs to the affective interpretation, gross inaction or fleeting audience engagement will result in characteristically negative affective interpretation in some dimensions of the PAD model (most often arousal and dominance).

This has implications for the identification of user engagement and flow-like properties, where the characteristic affective interpretations of user interaction (or inaction) are important, and manifest themselves as patterns of affective response.

One of the specific advantages of the eTree installation at an experimental level is that the affective multimodal interface can be used both for the artistic purpose of the installation (of which it is a component, since the installation itself should react to users' affective behaviour) and as a research tool, hence making affective measurements unobtrusive. In addition, this type of installation, because of its responsive nature, actually prompts user interaction through appropriate feedback, without the need to brief the users/spectators about the interaction modes (encouraging the exploratory behaviours already mentioned).

Furthermore, having users interact in pairs with the installation during our evaluations significantly increased use of speech (through questions and comments) thus ensuring significant input through that modality.

\section{FLOW AND THE INTERACTIVE ART EXPERIENCE}

The original concept of Flow [9] was involved with achieving optimal (i.e., most enjoyable) experiences in work and leisure situations, where enjoyment is derived from activities that are challenging and require an element of skill. This is distinct from simply experiencing pleasurable sensations, and encompasses the sense of novelty and accomplishment by facing and mastering the unexpected, of achieving something unique.

Outside of work-related activities, this most naturally finds an application in leisure activities that are skilful in nature, such as sports and games, and creative arts. Here, opportunities abound to refine and apply skills, explore the unknown (the raw material for an artwork), or face the challenges of an equally skilful opponent (in sports or competitive games).

Relating flow to experience of artistic media (rather than its creation) requires recognition of the structures and challenges inherent in the interpretation process [27,12]. If this is the case,

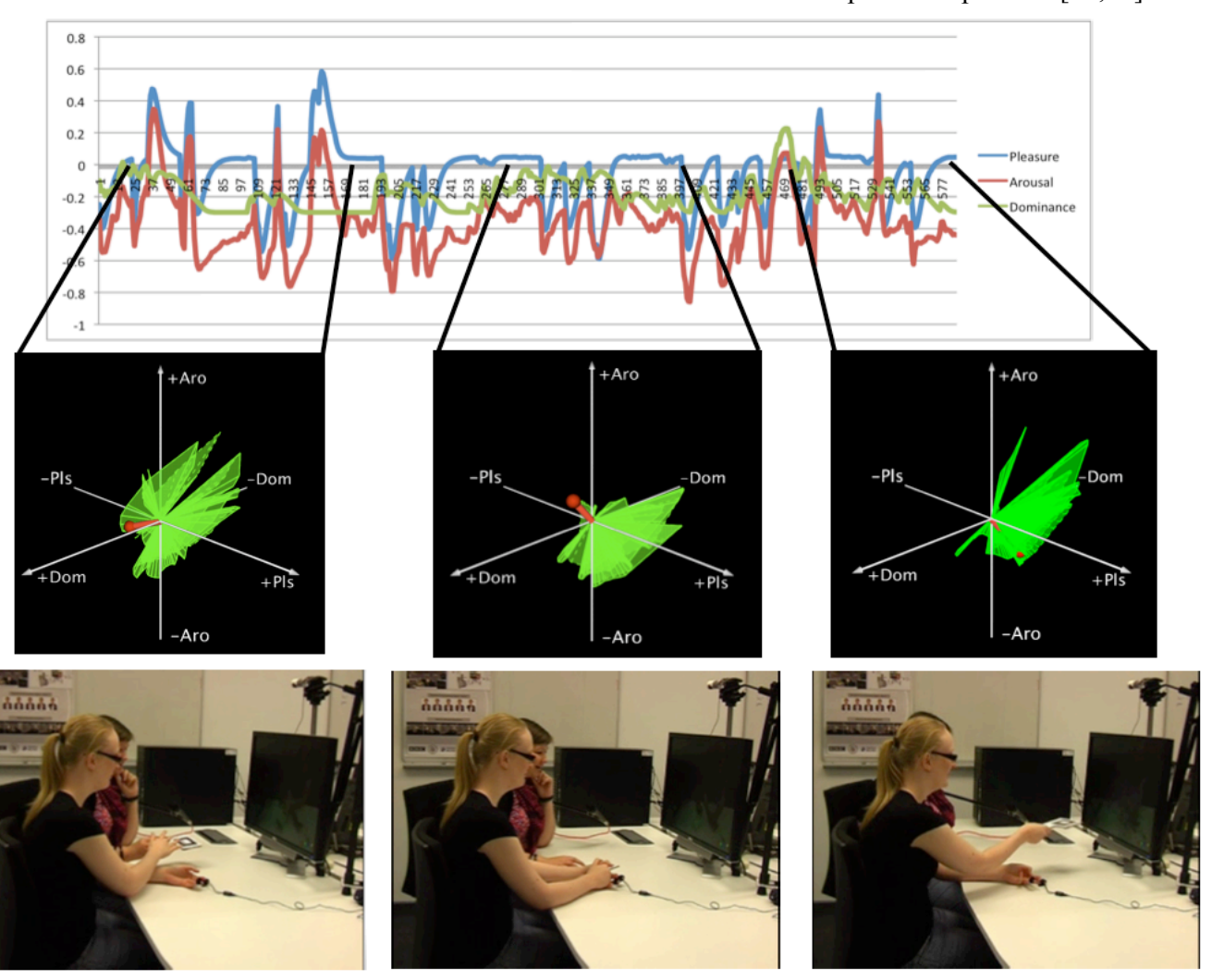

Figure 3. Affective dynamics of an e-Tree session. 


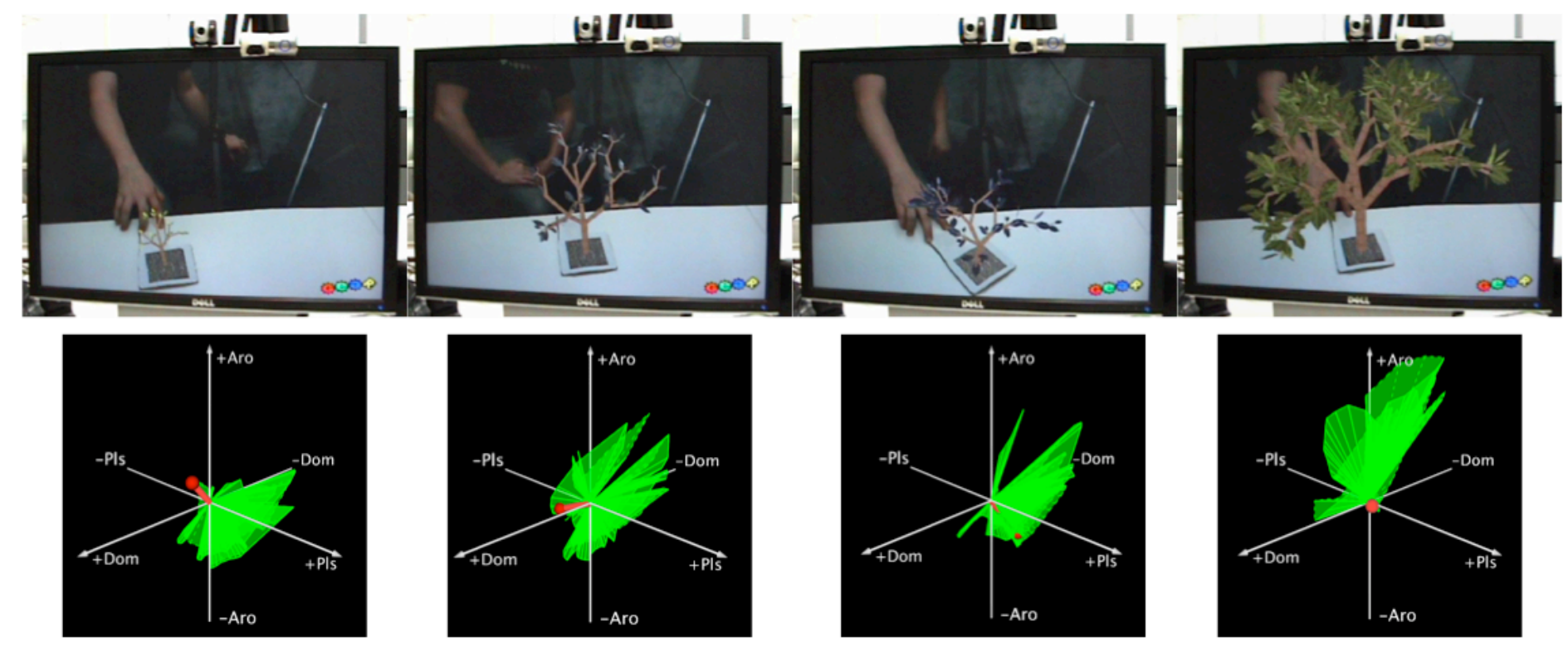

Figure 4. Development of e-Tree artwork.

then some examples, and possibly whole types, of art and media will be more inducing of a flow experience than others. The enjoyment of media as a flow activity is also argued by Sherry [33], who nonetheless posits videogames as a canonical example of interactive new media.

While Manovich [23] claims a psychological interactivity that is characteristic of all media, not just the so-called new one, there is still a gap between the physicality of sports and most games (and the flow experiences thereof) and the psychological relationship with experience of traditional media. The ability to channel ones skills into affecting the outcome of an experience is important for flow when that experience is interactive in nature. This stems from the inherent "self-as-cause" of the flow experience [12]- the psychological interactivity of media enjoyment is by definition internal to oneself, while a physical experience requires the ability to effect causes externally.

This physical interactivity is considered by some to be the "true" interactivity central to new media [30], with the narrative aspects, generally accepted as more related to content than interaction, taking a subservient role. Here, the "rules" that define constraints and demand understanding are the ones that govern the interaction - the exploration of possible interaction becomes the goal of the experience.

Computer systems in of themselves entail this interactivity, due to the user interfaces involved. Accordingly, models of flow have evolved that consider the environments supported by computerbased technology, such as the web [28,7], and video games [26, $8,36]$. Interactive art straddles these two concepts - the artistic narrative or metaphor remain in place as a first-class property, yet the experience itself is mediated by the physical interactions, interpreted by a computer system. Thus flow stems from both the challenges of artistic interpretation and of discovering and mastering affordances of interaction.

\section{MULTIMODAL DETECTION OF AFFECTIVE STATES}

The E-Tree interactive artwork incorporates a real-time representation of spectator affect that is generated by an affective multimodal fusion system which analyses multiple users' movements and speech for emotional content, and combines it into a single overall representation of the on-going affective experience. This is then used to influence the behaviours and appearance of the emotional tree as it grows and develops. We utilise this system to demonstrate that indicators of flow-like states can be measured and related to a real-time representation of affective state.

The emotional input of each affective modality in the system is represented by a vector in the PAD 3-D emotional space. These individual vectors are combined by a weighting algorithm to produce an overall vector representing the current affective state of the experience. As emotional input is analysed over the course of interaction with the artwork, the PAD vector is dynamically updated to track the changing representation of the affective state. This is illustrated in figure 3 , which shows the characteristic trajectories of the PAD vector for three sections of a typical interactive session with the E-Tree.

We consider this changing representation to stand for the user experience in two respects. Firstly, the regions of PAD emotion space that are encountered over the course of an experience reflect participants' overall emotional response to an interactive work. Secondly, that the dynamics of the changing PAD vector characterise the interactions and core essence of an interactive experience, and allow different instances of experience to be analyses and compared in a meaningful way.

As we have previously described [17], the affective multimodal fusion system generates a PAD vector representation for a number of affective modalities, including speech, physical interactions and movement and interest detection. Each PAD vector is 
calculated in each modality by mapping properties of feature analysis of a modality's to a combination of the PAD dimensions.

The PAD values of the overall vector are sent to an L-system generation component that maps each dimension of the PAD emotional model to parameters controlling the growth and appearance of the tree. The thickness of branches, size and colour of leaves and "droop" of the tree reflect the immediate affective state of the installation, while the growth and branching of the tree serve to record a history of the user experience, as it changes over time. The tree has a number of behaviours, such as "fading" that are triggered and controlled by the ongoing affective input. Examples of tree appearance after representative periods of affective input are shown as 3-D PAD trajectories in figure 4.

\section{FROM REAL-TIME AFFECTIVE MODELS TO FLOW}

Flow is often modelled with "channel segmentation models" that characterize different experiences in terms of the combination of challenge and skill. This was originally conceived as a state of flow occurring whenever perceived challenges were matched with personal skills [9], as shown in Figure 5a). Csikszaentmihalyi later refined this so that rather than reflect increasing ability within a particular task, it represented the day-to-day experiences, so that a flow state in any particular task would only be manifest if the challenges and skills were above typical levels [9], the state of low skill and challenge being labelled as "apathy". This model is shown in Figure $5 b$ )

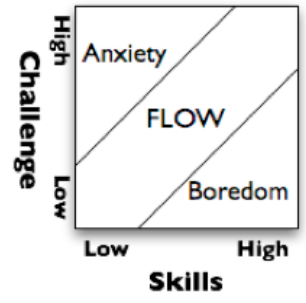

a)

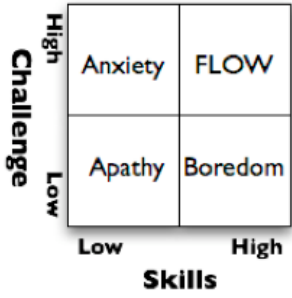

b)
Figure 5. Channel segmentation flow models.

The "channels" in these models contrast the positive flow state with negative states of anxiety, boredom and apathy depending on the relative levels of challenges and skills.

Affect has been posited as an indicator of flow [14] and studies have indicated that positive measures of affect are associated with flow experiences [22]. The labelling of the non-flow channels with affect-related words and the conception of flow as enjoyment further indicates the relationship between the model of flow and the affective aspects of an experience.

We posit that is not clear exactly how one would define the "challenges" provided by an interactive artwork (that does not of itself provide a goal-oriented framework, like a game), or the progression of skills developed by users, especially over the shortterm interactive sessions that would typically make up an experience with the artwork. Yet, we are still interested in notions of flow as a reflection of user experience. We propose that how to utilize an appropriate model of affect in order to detect emotional indicators of flow (and non-flow) channels.

\section{Flow Channels and Affect}

The PAD affective space can be divided into regions depending on whether each dimension is positive $(+\mathrm{P},+\mathrm{A},+\mathrm{D})$ or negative $(-$ $\mathrm{P},-\mathrm{A},-\mathrm{D})$. We will use these broad divisions to relate flow channels to affective representations (using mappings of affective terms derived by Mehrabian to justify dimensional values).

The enjoyable flow state has been associated with positive measures of affect, so we associate it with the $(+\mathrm{P},+\mathrm{A},+\mathrm{D})$ octant of the PAD model-pleasurable, aroused and in control (which tallies with Csikszentmihalyi's description of enjoyment). The non-flow channels are all labelled with negatively valenced affective terms, (-P). This suggests that in this model that Pleasure is a consequence of being in flow vs. experiencing conditions that do not facilitate flow. It is not clear that pleasure is required for a flow experience, or if pleasure alone can be a determinate for flow (which we shall discuss later).

The other dimensions of the PAD model (Arousal and Dominance) are more easily relatable to the flow channel model. Dominance relates to control and influence, so we consider states where there is an adequate level of skill for the challenge to engender positive dominance $(+D)$, and inadequate skill to foster negative dominance (-D). This would allow dominance to serve as a proxy measure of the relative level of skill without having to precisely define skills and challenges. Arousal $(+\mathrm{A},-\mathrm{A})$ can be related to how stimulating an experience is, with greater and varying challenges provoking more arousal. This means that an arousing (and therefore relatively challenging) experience can be either flow-like $(+\mathrm{A},+\mathrm{D})$ or anxious $(+\mathrm{A},-\mathrm{D})$ depending on how in control a user feels (due to their relative skill level), or in other words, whether they are in a dominant or submissive affective state.

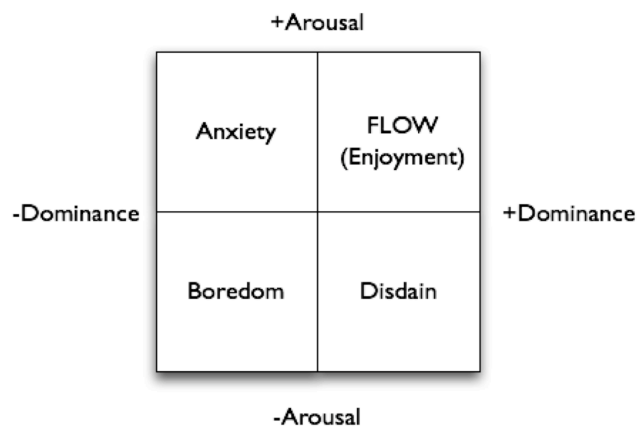

Figure 6. Affective mapping of channel flow model.

Low arousal (engendered by unchallenging conditions) experiences lead to either boredom or apathy in the flow channel model depending if relative skill level is high (boredom) or low (apathy). In terms of the PAD model, these two terms lie in the same region (-P,-A,-D). From our mapping of dominance to skill level, relative low skills would correspond to submissiveness, which could be characterized by either apathy or boredom, and relative high skills would indicate a dominant state $(-\mathrm{P},-\mathrm{A},+\mathrm{D})$, which would be more appropriately termed as dismissive or disdainful. We feel that this still fits with the channel model, but that the selection of affective terms is misleading. We instead propose that boredom/apathy $(-\mathrm{P},-\mathrm{A},-\mathrm{D})$ represent low challenge 
and skill, while disdain $(-\mathrm{P},-\mathrm{A},+\mathrm{D})$ represent low challenge and high skill.

Our re-formulation of the 4-channel model in terms of Arousal and Dominance using these terms is shown in Figure 6.

\subsection{Pleasure and Flow}

In can be seen that non-flow channels are labelled with negatively valenced terms, (-P) while flow is considered positively valenced $(+\mathrm{P})$. This leads to two questions about the relationship of affective state and flow. Firstly, which is the nature of those affective states that are pleasurable $(+\mathrm{P})$, yet either relaxing $(-\mathrm{A})$ or submissive (-D)? Secondly, what is the nature of the affective state that is both arousing and dominant $(+\mathrm{A},+\mathrm{D})$, yet displeasing $(-\mathrm{P})$ ?

Consideration of these regions of affective space may give further insight into non-flow states that can be recognized in order to help create conditions in which flow can be achieved.

A pleasurable, aroused yet submissive state $(+\mathrm{P},+\mathrm{A},-\mathrm{D})$, could be characterized as being "impressed" or "dependent"-where the imbalance of challenge and skill engenders awe, motivation or determination rather than anxiety. Pleasurable, relaxed and dominant states $(+\mathrm{P},-\mathrm{A},+\mathrm{D})$ can indicate the pleasure derived from low-challenge situations, such as watching TV or playing a relaxing game, where rather than being engrossed in the experience itself (as in a flow experience), the mind is able to "wander". Finally, a pleasurable, non-aroused and submissive state $(+\mathrm{P},-\mathrm{A},-\mathrm{D})$ could exist when just starting out in an experience, where intrinsic motivation and the hope of an increased challenge overcomes the apathy/boredom of a lowchallenge/low-skill situation.

While pleasure is an important distinguishing feature between non-flow states, it is not clear if it is truly an issue when experiencing flow. A displeasurable, aroused and dominant affective state $(-\mathrm{P},+\mathrm{A},+\mathrm{D})$ is characteristic of disgust or anger, which are not dealt with in existing flow model. It is our guess that pleasure within a flow experience serves as feedback to the choices and actions of the user, and does not necessarily interrupt flow, but that a consistent displeasurable affective state (even in flow) might indicate that the flow state may be unsustainable and that action might need to be taken to sustain flow.

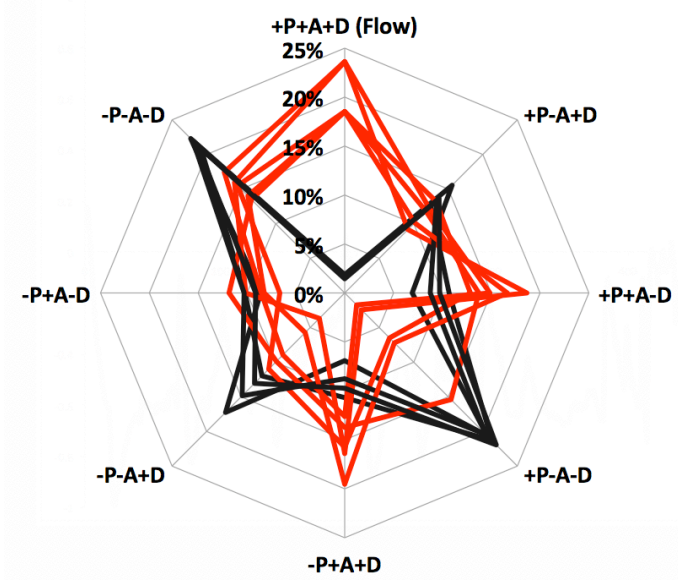

Figure 7. Characteristic PAD octant distributions.
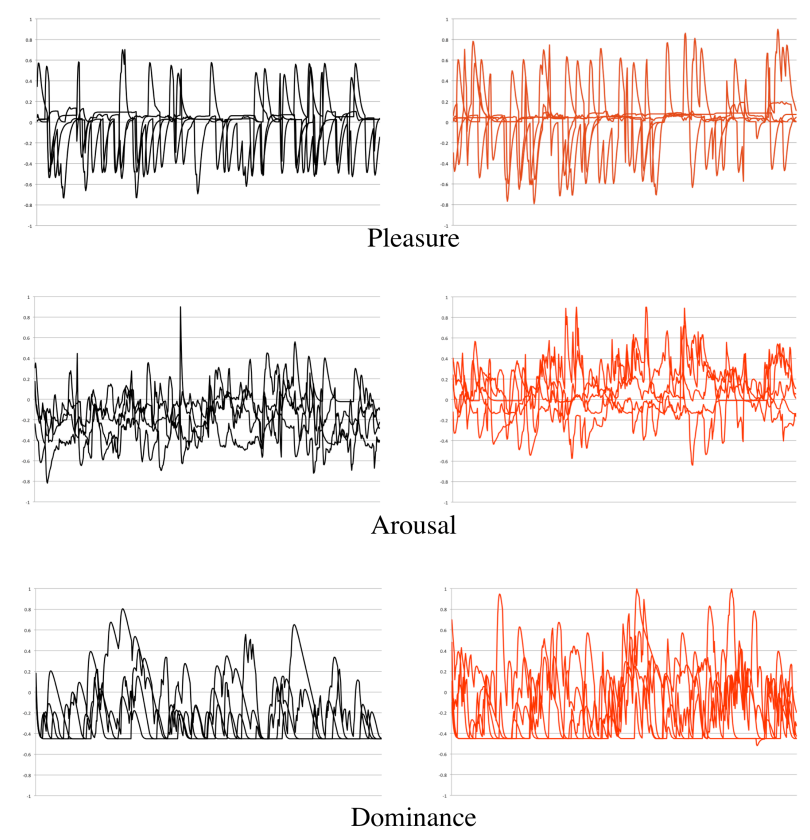

Figure 8. Dynamic PAD traces show similarity of interaction volume for unengaged (left) and engaged (right) participants.

\section{EVALUATION}

We have conducted two sets of initial user testing using the e-Tree interactive artwork, collecting real-time PAD values as described in the previous section, in an initial attempt to establish a link between flow and affective measurements. From the first set of tests (20 subjects, in 10 pairs), we identified two characteristic patterns of affective experience, based on the percentage of measured PAD in each octant over the course of an interactive session. (Figure 7.)

This illustrates well the overall relative levels of engagement, with a general increase in aroused and dominant states (associated with sustained interaction) being associated with reported higher levels of engagement. The group with a generally lower proportion of arousal and a majority of submissiveness reported lower subjective levels of engagement. The "engaged" group also had higher a significance percentage of affective input in the predicted "flow" octant of PAD space $(+\mathrm{P}+\mathrm{A}+\mathrm{D})$.

This reflects two trends in our interactive installation. Firstly, that engagement with the system leads to a higher proportion of aroused, dominant affective input, due to the interpretation of user interaction. This does not mean that the other octants are not entered, as can be seen in figure 8, which shows the similar volume of affective output for both engaged (right) and nonengaged (left) across a whole session for each PAD dimension. Both groups showed a similar range of affective input in all three dimension, and even non-engaged participants displayed emotional responses throughout the session, so their engagement (or lack of) is not down to major differences in the experience of the artwork (such as it not growing at all, or displaying no positive reactions.

The second trend is that while the less-engaged group appear more evenly spread throughout all octants (so are exhibiting a full range of affective response, when they do respond, albeit with 


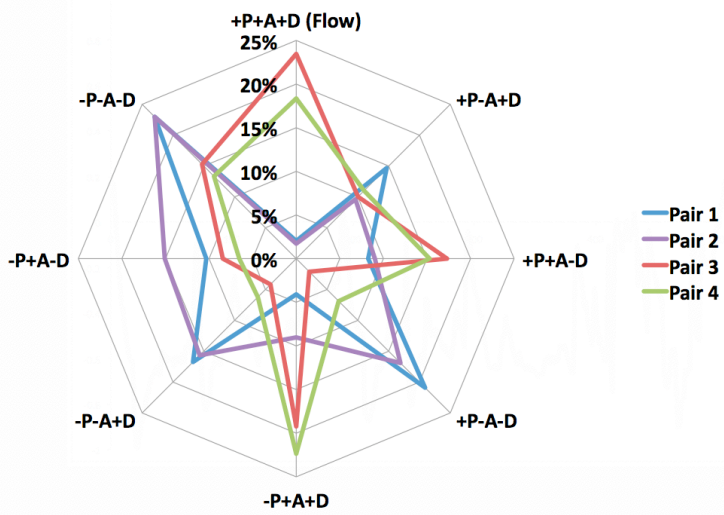

Figure 9. PAD octant distribution for flow test subjects.

barely any input in the $+\mathrm{P}+\mathrm{A}+\mathrm{D}$ or flow octant), the gross levels of affective input are lower, due to the lack of engagement. This again, can be seen in figure 8 , where median values of arousal $(0.3$ vs. -0.17$)$ and dominance $(0.13$ vs. -0.25$)$ are significantly different.

The second set of testing was a preliminary study where participants ( 8 subjects in 4 pairs) also answered a flow questionnaire after their interactive session. This was based upon the Flow State Scale [15], and consisted of questions about each element of flow experience as outlined by Csikszentmihalyi [8], presented as 7-level Likert scales. We have not analysed these scales for the influences of each item, but rather intend it as a naïve measure of flow to prepare for further study. An overall flow measure was obtained from the mean score across all the questionnaire items, normalized to give a value between 0.0 (no evidence of flow) to 1.0 (certain flow). The PAD measures for this group are shown in figure 9 , and as can be seen, they fall into the two patterns of affective state identified in the first study. Pairs 1 and 2 did not have engaging experiences, with little vocal reaction (and thus inhibiting the detection of emotion from voice), while pairs 3 and 4 were more engaged and exhibited more exploratory behaviour, with higher amounts of discussion between the participants.

Table 1 below shows the relationship between affective measures and selected flow questionnaire results. As expected, when a higher percentage of measure affect was in the positive octant of PAD space, flow scores were higher. In addition, Challenge scores were higher when a majority of affective input involved positive Arousal (+A) and Skill scores were higher for positive Dominance $(+\mathrm{D})$. This was calculated from the questionnaire items that related to challenges and skills, respectively.

Table 1. Flow measures and affective distribution.

\begin{tabular}{lllllll}
\hline & $+\mathrm{P}+\mathrm{A}+\mathrm{D}$ & Flow & $+\mathrm{A}$ & Chall & $+\mathrm{D}$ & Skill \\
\hline Pair 1 & $2.1 \%$ & 0.2 & $25 \%$ & 0.21 & $38 \%$ & 0.29 \\
Pair 2 & $1.7 \%$ & 0.36 & $35 \%$ & 0.14 & $36 \%$ & 0.21 \\
Pair 3 & $23 \%$ & 0.63 & $68 \%$ & 0.64 & $57 \%$ & 0.57 \\
Pair 4 & $18 \%$ & 0.62 & $63 \%$ & 0.57 & $58 \%$ & 0.57 \\
\hline
\end{tabular}

These results are encouraging and point towards a potential link between affective state measures and evidence of flow, which deserves further investigation. However, the number of subjects test so far is low, so we cannot comment yet on the statistical significance of the results.
For the E-Tree installation, it appears that when users are enthusiastic and engaged with the artwork, affective states are supported that are indicative of flow, whereas if they do not have a successful experience, affective states that would indicate flow are not able to be measured.

\section{CONCLUSIONS}

We have introduced a model of user experience that is based on a temporal extension of dimensional emotional models. In its philosophy, this model is compatible with recent research in (noninteractive) media content which describe the evolution of affective features through time on a linear axis [19], or as a trajectory in a tri-dimensional affective space, similar to PAD [3] Our representation is well adapted to an interactive context since the emotional model is instantiated from interaction channels. Our findings confirm those observed for non-interactive media, in the sense that user experience could be described through the trajectory dynamics of through its distribution within the PAD space. Our intuition was that specific trajectory patterns, in particular along the arousal and dominance axes, could be related to phenomenological models such as Flow. This was actually confirmed by early results obtained from a comparison of PADspace trajectories and Flow questionnaires for several subject pairs. This model has the potential to describe aesthetic experiences even in the absence of a priori categories either for individual users or small groups of spectators.

\section{ACKNOWLEDGEMENTS}

This work has been funded in part by the European Commission under grant agreement CALLAS (FP-ICT-034800).

\section{REFERENCES}

[1] Benayoun. M., http://www.benayoun.com/works.php

[2] Benini, S. Canini, L., Migliorati, P. and Leonardi, R. 2009. Multimodal Space for Rushes Representation and Retrieval, In: Proceedings of the 7th International Workshop on Content-Based Multimedia Indexing (CBMI), Chania, Crete Island, Greece, pp. 50-55.

[3] Bernhaupt, R., Boldt, A., Mirlacher, T., Wilfinger, D. 2007. Tscheligi, M. Using emotion in games: emotional flowers. ACM SIGCHI Advances in Computer Entertainment Technology, pp. 41-48.

[4] Bilda, Z., Edmonds, E., and Turnbull, D. 2007. Interactive experience in a public context. In: Proceedings of the 2007 Conference on Creativity and Cognition, Washington DC, USA. pp. 243-244.

[5] Boden, M.A. (in press), Aesthetics and Interactive Art. In C. Makris, R. L. Chrisley, R. W. Clowes and M. A. Boden (Eds.) Art, Body, Embodiment, Cambridge Scholars Publishing: Newcastle.

[6] Cavazza, M., Lugrin, J., Hartley, S., Libardi, P. Barnes, M. J., Le Bras, M., Le Renard, M., Bec, L., and Nandi, A. 2004. New ways of worldmaking: the Alterne platform for VR art. In Proceedings of the 12th Annual ACM international Conference on Multimedia. MM '04. New York, 80-87.

[7] Chen, H., Wigand, R.T., Nilan, M.S. 1999. Optimal Experience of Web Activities. Computers in Human Behavior, 15 (5), 585-608.

[8] Chen, J. 2007. Flow in games (and everything else). Commun. ACM 50, 4 (Apr. 2007), 31-34. 
[9] Csikszentmihalyi, Mihaly. 1975 Beyond boredom and anxiety. Jossey-Bass Publishers, San Francisco.

[10] Csíkszentmihályi, Mihályi. 1990 Flow: The Psychology of Optimal Experience. New York: Harper and Row.

[11] Csikszentmihalyi M, Larson R., 1987. Validity and reliability of the Experience-Sampling Method. J Nerv Ment Dis. 1987 Sep;175(9):526-36.

[12] Csikszentmihalyi, M. and Robinson, R.E. 1990. The Art of Seeing: An Interpretation of the Aesthetic Encounter.J. Paul Getty Museum Publications, LA, ISBN: 0892361565.

[13] Edmonds, E., Everitt, D., Macaulay, M., Turner, G. 2004. On physiological computing with an application in interactive art, Interacting with Computers, Volume 16, Issue 5, Physiological Computing, October 2004, Pages 897-91.

[14] Ellis, G.D., Voek1, J. E. \& Morris, C. 1994. Measurement and Analysis Issues with Explanation of Variation in Daily Experience Using the Flow Model. Journal of Leisure Research 26(4), 337-356. National Recreation and Park Association.

[15] Fishwick, P.A. (Ed.), 2006. Aesthetic Computing. Cambridge, MIT Press.

[16] Gilroy, S. W., Cavazza, M., Chaignon, R., Mäkelä, S., Niranen, M., André, E., Vogt, T., Urbain, J., Billinghurst, M., Seichter, H., and Benayoun, M., 2008. E-Tree: emotionally driven augmented reality art. In Proceedings of the 16th ACM international Conference on Multimedia (MM '08). ACM, New York, NY, 945-948.

[17] Gilroy, S. W., Cavazza, M., Chaignon, R., Mäkelä, S., Niranen, M., André, E., Vogt, T., Urbain, J., Seichter, H., Billinghurst, M., and Benayoun, M., 2008. An affective model of user experience for interactive art. In Proceedings of the 2008 international Conference on Advances in Computer Entertainment Technology (Yokohama, Japan). ACE '08, vol. 352. ACM, New York, NY, 107-110.

[18] Grau, O. 2004. Virtual Art: From Illusion to Immersion. MIT Press. ISBN 0-262-57223-0.

[19] Hanjalic, A. 2006. Extracting moods from pictures and sounds. IEEE Signal Processing Magazine 23, 2 (March 2006), pp. 90-100.

[20] Helfenstein, S. 2005. Product Meaning, Affective use and Transfer. Human Technology, 1, 76-100, April 2005.

[21] Jackson, Susan A., 1996. Toward a conceptual understanding of the flow experience in elite athletes. Research Quarterly for Exercise and Sport, 67(1), 76.

[22] Mannell, R., Zuzanek, J., Larson R., 1988. Leisure states and flow experiences: Testing perceived freedom and intrinsic motivation hypotheses. Journal of Leisure Research 20. 289304.

[23] Manovich, L., 2001. The Language of New Media. The MIT Press, Cambridge, Massachusetts.

[24] Mehrabian, A., 1996. Pleasure-arousal-dominance: A general framework for describing and measuring individual differences in temperament. Current Psychology:

Developmental, Learning, Personality, Social, 14, 261-292.

[25] Morrison, A., Mitchell, P., Brereton, M., 2007 The Lens of Ludic Engagement: Evaluating Participation in Interactive Art Installations. ACM Multimedia 2007, Augsburg, Germany, pp. 509-512.

[26] Nacke, L. and Lindley, C. A. 2008. Flow and immersion in first-person shooters: measuring the player's gameplay experience. In Proceedings of the 2008 Conference on Future Play: Research, Play, Share (Toronto, Canada). Future Play '08. ACM, New York, NY, 81-88

[27] Nell, V. 1988. The Psychology of Reading for Pleasure: Needs and Gratifications Reading Research Quarterly, Vol. 23, No. 1 (Winter, 1988), pp. 6-50.

[28] Novak, T. P., Hoffman, D. L., Duhachek, A. 2003. The Influence of Goal-Directed and Experiential Activities on Online Flow Experiences. Journal of Consumer Psychology, Vol. 13, No. 1/2, Consumers in Cyberspace (2003), pp. 3-16. Lawrence Erlbaum Associates

[29] Picard, R. Affective Computing, 1997. Cambridge (Mass.), MIT Press.

[30] Polaine, A., 2005. The flow principle in interactivity. In Proceedings of the Second Australasian Conference on interactive Entertainment, ACM International Conference Proceeding Series, vol. 123. Creativity \& Cognition Studios Press, Sydney, Australia, 151-158.

[31] Popper, F., 2007. From Technological to Virtual Art. Cambridge (Mass.), MIT Press.

[32] Porat, T., Liss, R., Tractinsky, N., 2007. E-Stores Design: Influence of E-Store Design and Product type on consumers' emotions and attitudes. 12th International Conference, HCI International 2007, Beijing, China, Proceedings Part IV, LNCS 4553, pp. 712-721.Springer-Verlag.

[33] Sherry, J.L. 2004. Flow and Media Enjoyment. Communication Theory 14(4), pp. 328-347.

[34] Shugrina, M., Betke, M., Collomose, J.P. 2006. Empathic Painting: Interactive stylization using observed emotional state. Proceedings 4th Intl. Symposium on Non-photorealistic Rendering and Animation (NPAR 2006), pp. 87-96. ACM Press.

[35] Sommerer, C. and Mignonneau, L., 1999. Interactive Plant Growing. In Ars Electronica - Facing the Future, Cambridge, MA: MIT Press, 393-394.

[36] Sweetser, P. and Wyeth, P., 2005. GameFlow: A Model for Evaluating Player Enjoyment in Games. In ACM Computers in Entertainment, 1(3).

[37] Vorderer, P. Entertainment theory, 2003. In J. Bryant, D. Roskos-Ewoldsen, \& J. Cantor (Eds.), Communication and emotion: Essays in honor of Dolf Zillmann Hillsdale, N.J.: Lawrence Erlbaum Associates, , 131-153.

[38] Watanabe, T, 2007. Human-Entrained E-COSMIC: Embodied Communication System for Mind Connection. HCI International 2007, Beijing, China,1008-1016. 